\title{
Numerical Process Modelling and Simulation of Campbell Running Systems designs
}

Chengcheng Lyu, Michail Papanikolaou and Mark Jolly

Manufacturing Department, Cranfield University, Cranfield, MK43 0AL, UK

\begin{abstract}
In the 1980s John Campbell developed a new casting process from his research in the industry over a number years. The Cosworth Process was for delivering very high quality aluminium components for the automotive industry. The process was very capital intensive and not very flexible for smaller companies delivering lower volumes of product. However, the principles behind the process have been taken and used to develop a range of different so-called "running systems" to help improve the quality of castings. Some of these designs have been published in a "Castings Handbook" [1] authored by Campbell. This paper presents the results of an MSc project during which a number of the proposed designs from Campbell's Mini Casting Handbook [2] for certain features in running systems have been modelled using a validated CFD software.
\end{abstract}

Keywords: Gravity sand casting, Running system design, Numerical simulation, Vortex gate, Trident gate, Bubble trap, Multiple-in-gate, Air entrainment, Gate velocity.

\section{Introduction}

In order to add control to the whole casting process a set of rules has been proposed by Campbell commonly known as the "10 Rules of Casting" ([3], [4]). These rules have been applied to a whole range of alloys including aluminium, zinc, copper and cast iron. A poorly designed running system contributes to most of the defects of the final casting as most of the damage occurs in this region [1]. That is because all metal into the cavity has to go through the running system first. Moreover, the very beginning seconds of casting (the priming stage) is the most dangerous period of generating various defects, such as air entrainment, inclusion or misruns [5]. The problem of gravity filling is extremely critical because gravity naturally accelerates the liquid metal flow and consequently causes damage if not controlled properly. Campbell [1] has summarized five characteristics of a good running system. Namely they should:

1. be lightweight. This means the ratio of finished casting weight to total cast weight should be as high as possible;

2. control the velocity of metal into the casting which should be as low as possible, preferably, under the critical value;

3. avoid entraining any impurities into the system (such as slag, air or sand);

4. eliminate surface turbulence;

5. be easy to remove. It is better to avoid complicated curves so that breaking off the mould is simple.

While Campbell's description is more systematic, some other researchers proposed that a good running system should fill quickly (but tranquilly) and should boost directional solidification [6]; or should solidify quickly and uniformly [7]. Regardless of solidification process, among these different expressions, one core principle is believed to be similar; that the level of surface turbulence is the fatal factor for the quality of the casting.

It is noticeable that casting rule 2 and characteristic 2 both emphasise the importance of the critical gate velocity being less than $0.5 \mathrm{~ms}^{-1}$ and this does not vary much for different alloys while exceeding this value triggers surface turbulence and the consequent defects. However, this value is extremely easily violated for a gravity casting system because the critical velocity $0.5 \mathrm{~ms}^{-1}$ for an aluminium alloy is merely equivalent to a drop of about $12.5 \mathrm{~mm}$. This is almost impossible to achieve in a gravity poured system except possible in an open shallow mould.

To address the conflict of necessary fall height and the rigorous requirement of low velocity for a closed mould techniques must be developed to enable a low velocity before metal enters the ingates. And in fact, after deep understanding of the importance of this critical value, efforts have always been made by researchers and founders to get close to it.

Earlier researchers proposed that expansion in cross section helps reduction of velocity, and this is the fundamental design principle of the so-called 'unpressurised system'. This is theoretically right according to the continuity equation. However, this type of system has been suspected to be unable to ensure good casting quality because of more likely air entrainment and vortex flow. This has been proven by some researchers through both simulation and experiment [8]. Researchers also tried to deal with the turbulent metal flow after it is poured into the pouring basin. According to Campbell, abandoning the conical basin should be the first step to take because of its extraordinary ability to generate an air guzzling vortex [9] (which Campbell described as an 'air pump'). While air is the most evil enemy of good castings, especially for alloys prone to active reaction with oxide, such as aluminium, titanium and magnesium alloys, the reason that the conical basin is still in use is because of its cheap and practical application. It is shocking that in the investment casting sector, who claim to have the highest added value and premium casting process, the majority still use a conical pouring basin. Campbell proposed different shapes of offset pouring basin in his handbook [1], without step, with sharp step and with a radiused step. The latter is the one chosen for all the running systems in this research.

Rather than endlessly struggle to eliminate turbulence in the pouring basin, which may end up in achieving little, a technique named 'contact pouring' is strongly advocated by Campbell. This is achieved by using a small bottom pour ladle, usually capable of holding 10 to $20 \mathrm{~kg}$ metal, and is topped up from beneath the surface by a robot dipping into the metal. A stopper plugs the hole in the base of the ladle and when the ladle is full it is moved to contact the sprue and the 
stopper raised allowing the metal to enter the system. Combined with a well-designed downsprue this can deliver turbulence and oxide free molten metal into the system [10]. The challenge of this technique is to align the exit of the ladle to the entrance of the sprue and ensure reliable seal to avoid leakage and a passage for air entrainment.

After the metal enters the system, to reduce its velocity, filters are explored by simulation and experiments [11],

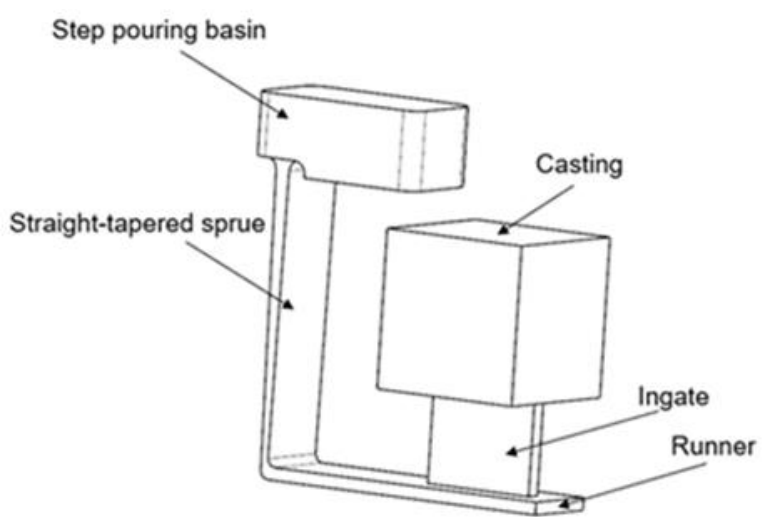

Figure 1: The basic running system (BRS)

Table 1: Basic Running System design dimensions

\begin{tabular}{ll}
\hline \multicolumn{1}{c}{ Parameters } & \multicolumn{1}{c}{ Value } \\
\hline Initial volume flow rate $\mathrm{Q}_{0}$ & $382 \mathrm{~cm}^{3} \mathrm{~s}^{-1}$ \\
Depth of pouring basin $\mathrm{H}_{\mathrm{b}}$ & $50 \mathrm{~mm}$ \\
Width of pouring basin $\mathrm{W}_{\mathrm{b}}$ & $65 \mathrm{~mm}$ \\
Length of pouring basin $\mathrm{L}_{\mathrm{b}}$ & $120 \mathrm{~mm}$ \\
Entrance area of sprue $\mathrm{A}_{2}$ & $590 \mathrm{~mm}^{2}$ \\
& $(59 \mathrm{~mm} \times 10 \mathrm{~mm})$ \\
Height of the sprue $\mathrm{H}_{\mathrm{S}}$ & $200 \mathrm{~mm}$ \\
Exit area of sprue $\mathrm{A}_{3}$ & $207 \mathrm{~mm}$ \\
& $(53 \mathrm{~mm} \times 4 \mathrm{~mm})$ \\
Area of runner $\mathrm{A}_{4}$ & $424 \mathrm{~mm}$ \\
& $(53 \mathrm{~mm} \times 8 \mathrm{~mm})$ \\
Length of runner $\mathrm{L}_{\mathrm{R}}$ & $200 \mathrm{~mm}$ \\
Width of ingate $\mathrm{W}_{\mathrm{in}}$ & $10 \mathrm{~mm}$ \\
Length of ingate $\mathrm{L}_{\mathrm{in}}$ & $75 \mathrm{~mm}$ \\
Height of ingate $\mathrm{H}_{\mathrm{in}}$ & $60 \mathrm{~mm}$ \\
Casting size & $100 \mathrm{~mm} \mathrm{cube}$ \\
\hline
\end{tabular}

right angle bends are also suggested to be an effective way to reduce velocity, but they may incur some other problems like entrainment of air and oxide [12] Unfortunately, none of these methods is as ideal as counter-gravity gating systems such as the Cosworth or Crimson processes.

It is common practice to assess and optimize a newly designed running system, researchers or founders have used try various techniques, classified as direct observation, modelling by water analogy or computeraided simulation [6]. The first two trial-and-error methods require long times and high cost [13]. Since the first published work on numerical simulation in the late 1980s, many software packages have been developed for foundries and have been used increasingly [14]. Computational Fluid Dynamics (CFD) software is considered to be the most costeffective tool for both design and optimization of a running system [15]. The traditional 'trial-and-error' in the workshop therefore is transformed to the 'proof-of-concept' by computer [16]. A range of simulation software are available today for casting engineers with different features. For this paper FLOW-3D ${ }^{1}$ has been used to conduct simulations and FLOWSIGHT for post-processing.

\section{Running System Designs}

The aim of this paper is to assess the effectiveness of different running systems for gravity sand casting. To simplify calculation, the casting part is a very simple geometry: an aluminium cube with the length of $100 \mathrm{~mm}$. A Basic Running System (BRS) was devised using both a published nomogram [1] and the method described by Jolly [12], as illustrated in Figure 1. Table 1 gives the dimensions and values used for the BRS.

As well as the BRS nine other systems were simulated with different features which included the placement of filters, bubble trap, a vortex gate, a trident gate and four designs of multiple gates (Figure 2). These designs were taken from pages 46, 49, 54 and 55 of Campbell's Mini Casting Handbook [2]. Simulation was proved to be an effective tool as after optimizing the mesh none of the simulations took more than an hour to run. All the simulations were run assuming an A356 alloy with an initial pouring temperature of $735^{\circ} \mathrm{C}$. Predicted filling times ranged from $4 \mathrm{~s}$ to $6.6 \mathrm{~s}$ which is reasonable as the initial system was designed to fill in 5 seconds.

\section{Simulation results and analysis}

The results of the 10 systems were compared in three groups. In most cases Entrained Air Volume Fraction (EAVF) and velocity were tracked and used as comparative measure of the effectiveness of the systems. In this paper only a selection of the results are presented as detailed below:

1. EAVF results with BRS as a benchmark, compared with HPF, LPF as well as LPF_BT to examine the performance of filter and bubble trap (Figure 2 a-c);

2. Velocity results with BRS as a benchmark, compared with VGS and TGS to display how well the latter two work in terms of reducing velocity (Figure $2 \mathrm{~d}-\mathrm{e}$ );

\footnotetext{
${ }^{1}$ https://www.flow3d.com/
} 
3. EAVF results for four multiple-in-gate systems (SRS, TRS, CJ_S and CJ_C) compared with each other to explore the best design for delivering metal into 3 identical castings (Figure $2 \mathrm{f}-\mathrm{i}$ ).

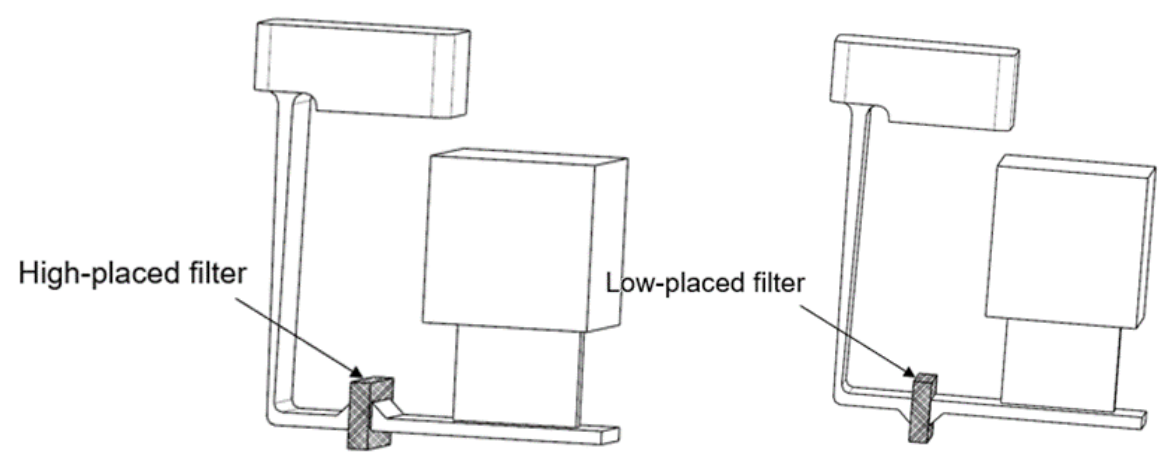

a) High Placed Filter (HPF)

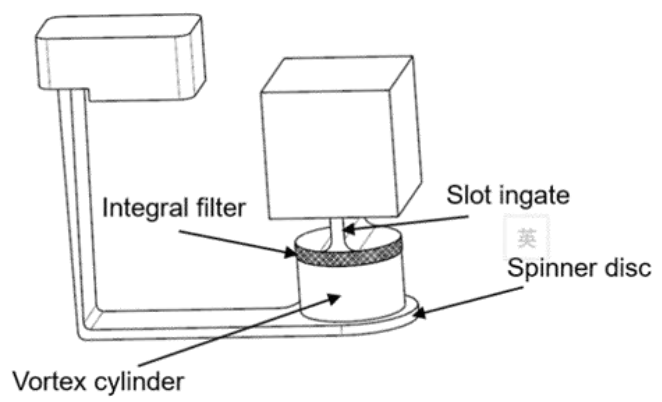

d) Vortex Gate System (VGS)

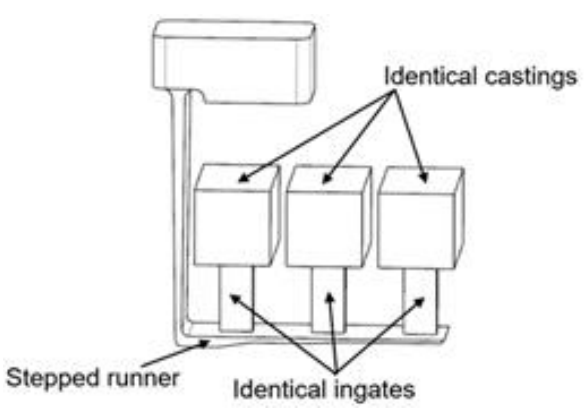

g) Stepped Runner System (SRS) b) Low placed Filter (LPF)

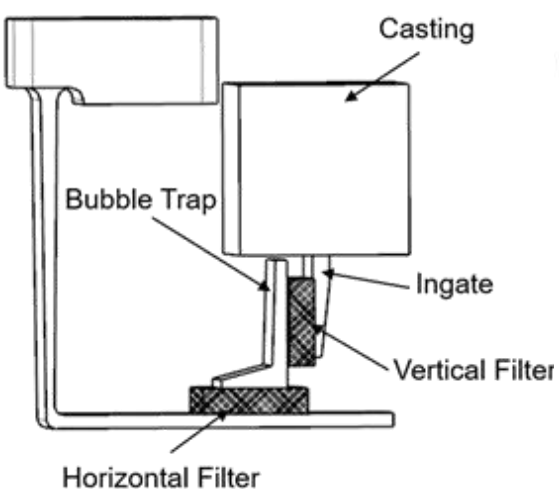

e) Trident Gate System (TGS)

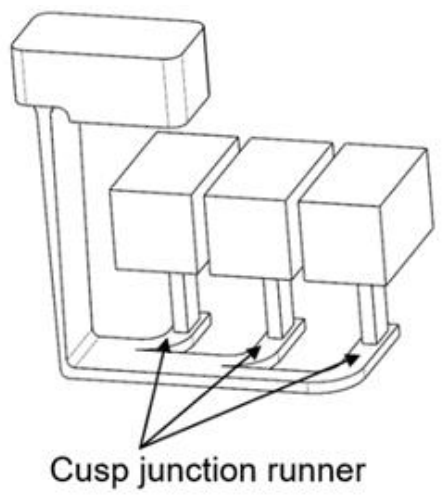

h) Cusp Junction Runner Straight (CJR_S)

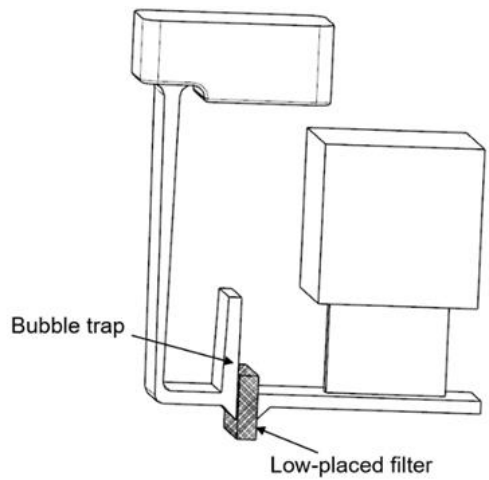

c) Low Placed Filter and Bubble Trap (LPF_BT)

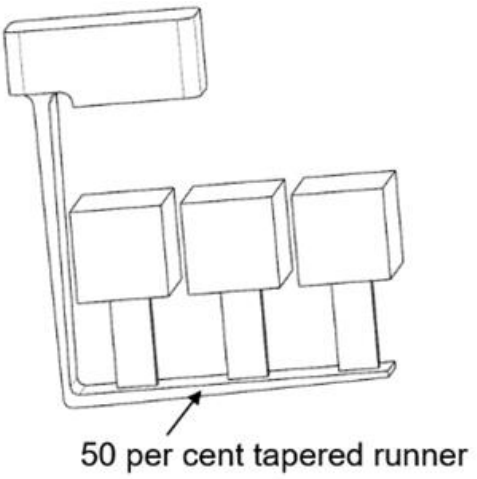

f) Tapered Runner System (TRS)

Figure 2: Isometric CAD images of the running system designs developed from features described in Campbell's Mini Casting Handbook [2] and used for comparative CFD simulations

Figures 3-5 are selected images from a range of simulations. Figure 3 shows a comparison of the predicted entrained air volume fraction (EAVF) for the basic running system (BRS), high placed filter (HFS), low placed filter (LFS) and low filter with bubble trap (LFS_BT). These comparisons are for the time at which the running system is close to being primed; in most cases just under $1 \mathrm{~s}$, and for the time when the casting has just filled (just over $4 \mathrm{~s}$ ). Figure 4 shows a comparison of the predicted metal velocities at $1.1 \mathrm{~s}$ and $3.3 \mathrm{~s}$ between the BRS and the vortex and trident gates. Particle tracking is included and shows the distribution of particles introduced into the pouring stream above the pouring basin. Figure 5 illustrates the effect that different multi-gate running system designs have on the EAVF at times early in the filling and just before the castings are filled. 
Time (s)

EAVF 0.00
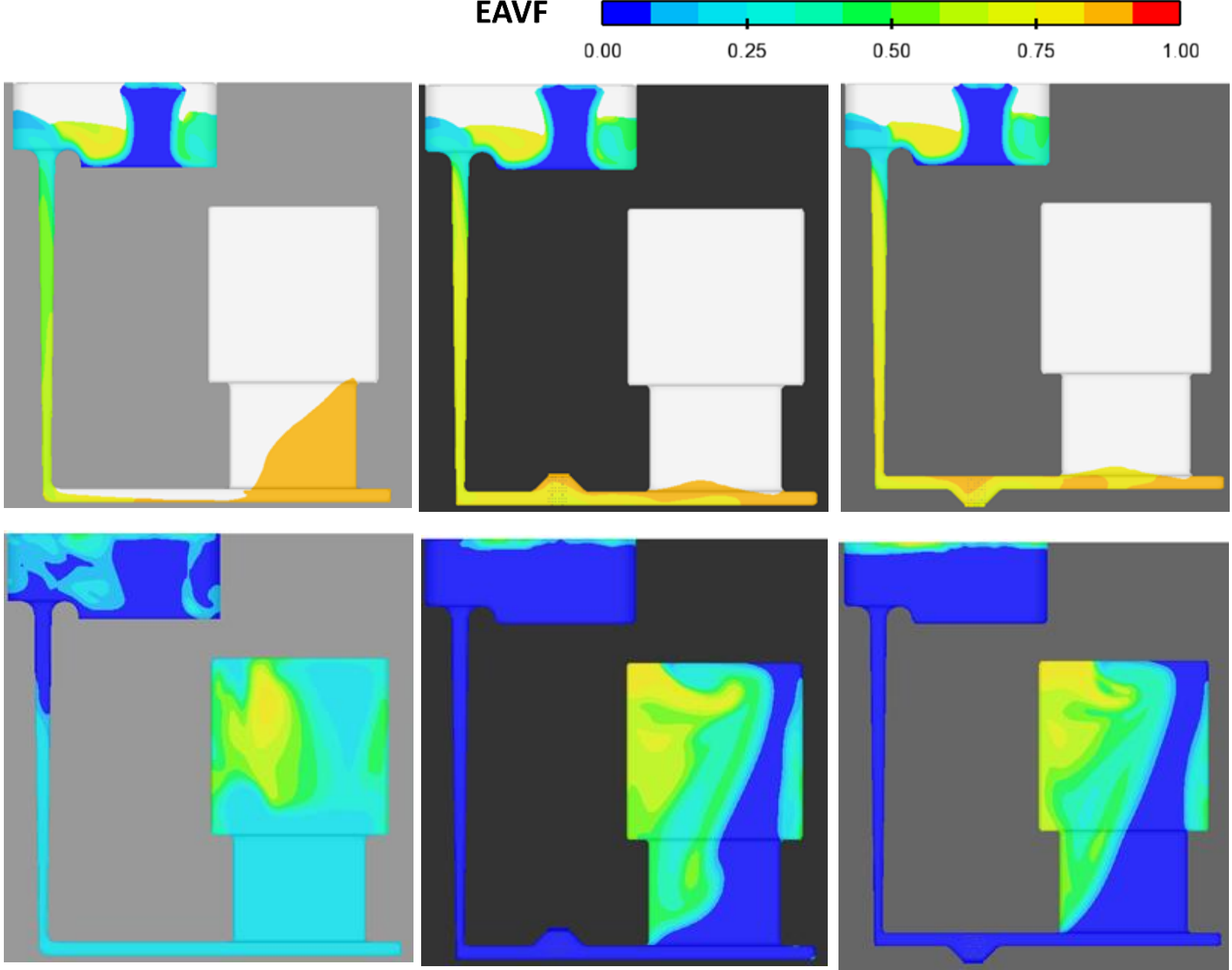
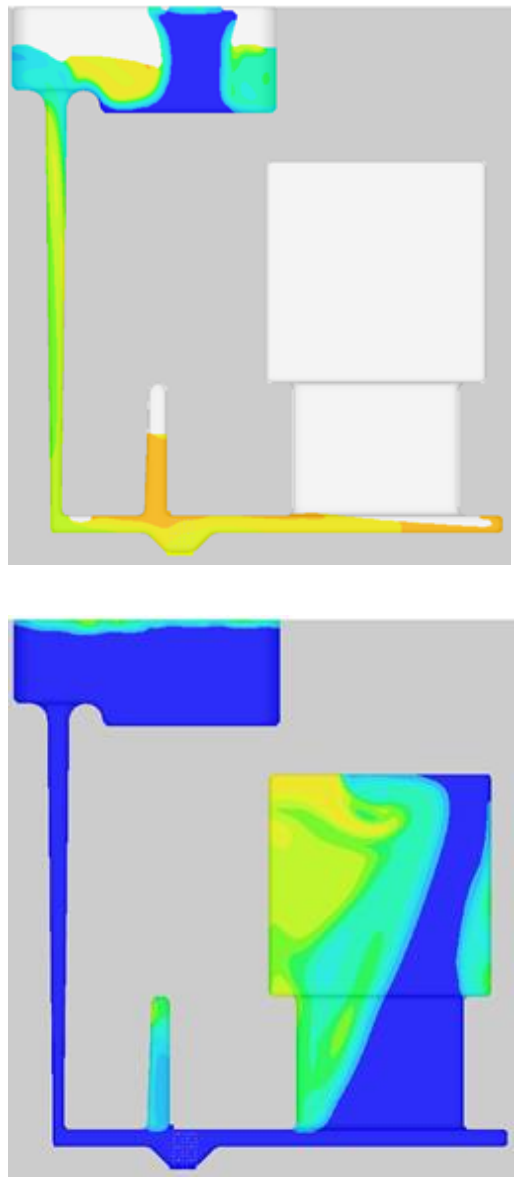

Figure 3: Predicted Entrained Air Volume Fraction (EAVF) results from the beginning and end of filling showing the effects of different filter placement and a bubble trap. Overall it can be seen that having a filter in the runner significantly reduced the amount of air entrainment by preventing a rolling back wave in the runner seen in the BRS. The bubble trap has a limited effect in this instance but does slightly reduce the air entrainment. 


\begin{tabular}{ccccc}
\hline 0.00 & 0.55 & 1.11 & 1.66 & 2.21
\end{tabular}
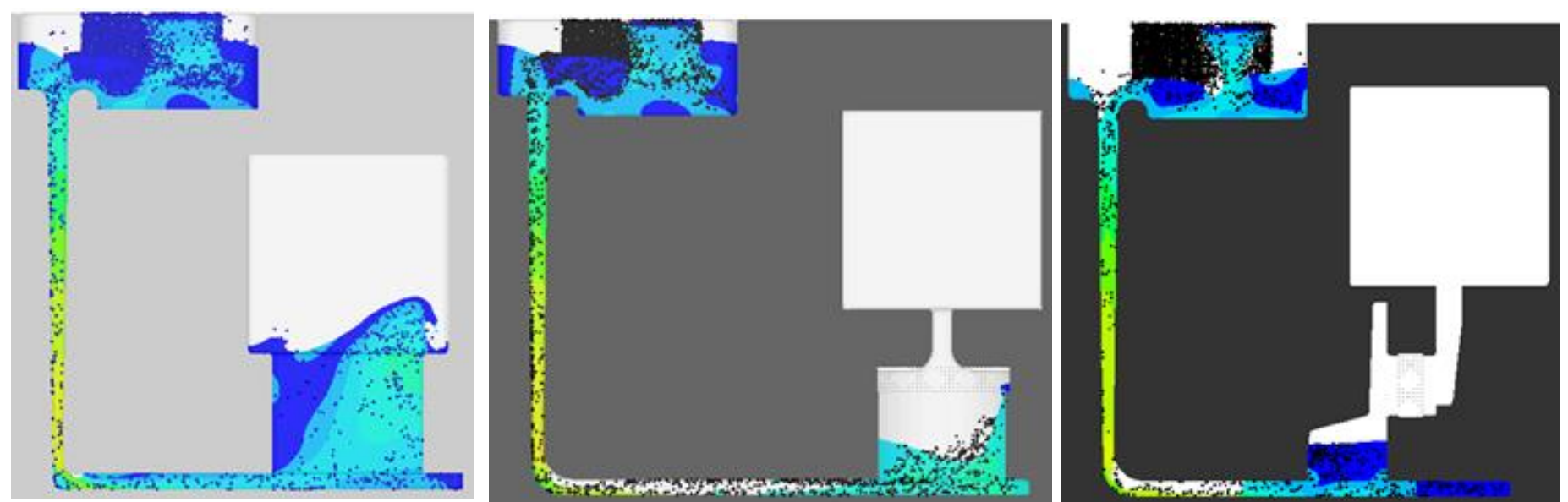

3,292
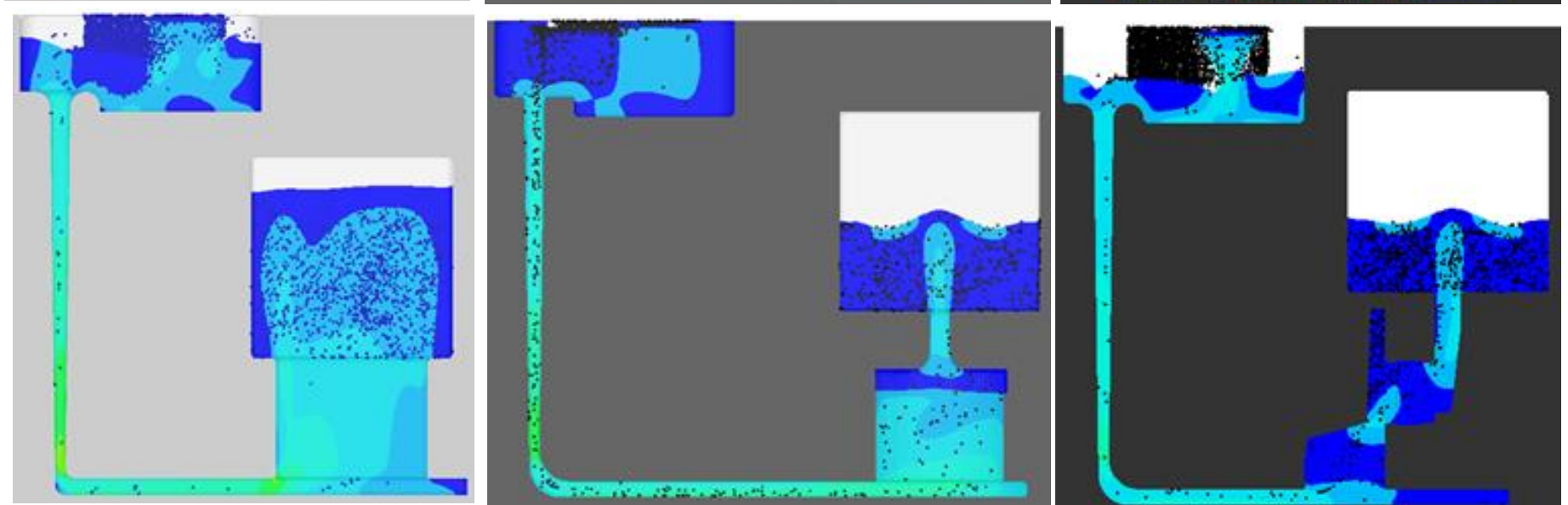

Figure 4: Comparison of Basic, Votex and Trident gates showing the considerable reduction in velocity achieved by the latter two designs. However, both the Vortex and Trodent gate showed much longer running sytem priming times with the Vortex gate being the slowest. The predicted filling tmes for the systems were 4.0, 4.95 and $6.59 \mathrm{~s}$ for the BRS, VGS and TGS respectively. 
0.50

0.75

1.00

0.914
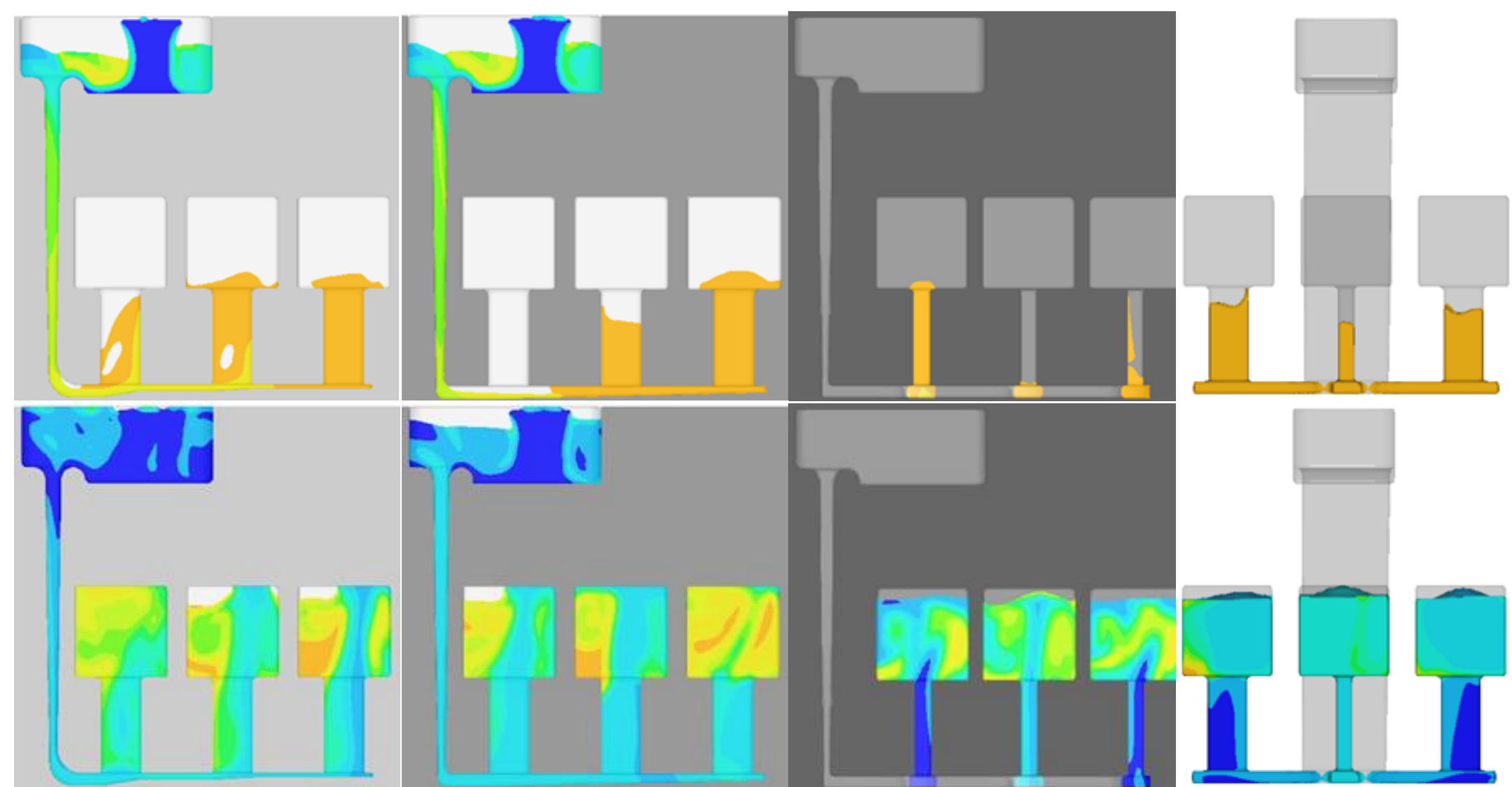

3.629

Figure 5: Predicted Entrained Air Volume Fraction (EAVF) for the 4 different multiple gate systems namely: stepped, tapered straight cusp and curved cusp clearly showing that the cusp systems are superior in as much as they appear to entrain less air than either the tapered or stepped runners. 


\section{Discussion of results}

Looking at Figure 3 it can be seen that the inclusion of a filter ensures that the running system primes quickly and in all cases faster than the BRS. At 0.885 the BRS is not primed and even though the runner bar is only $8 \mathrm{~mm}$ in depth there is clearly a rolling back-wave developing which would lead to surface oxide and air entrainment. It is clear that HPF, LPF and LPF_BT all work well in reducing EAVF comparing with BRS. After $4.12 \mathrm{~s}$ the castings are filled and the filtered castings show a much lower lever of air entrainment than the BRS. The bubble trap does appear to work as it has a higher level of air entrainment than the runner bar although the detailed analysis of this can only really be seen in the full simulations. Unrestricted jetting at the back of filter in HPF system is not observed as is detailed in the literature but this may be because of the method for modelling the filter which just applies a permeability across a volume representing the filter. Jetting would only be observed in a simulation if the actual physical geometry of the filter was modelled. [17]

Figure 4 demonstrates clearly the damping effect of both the vortex and trident gates on the velocity of the liquid metal compared with the BRS. Both these gating systems have filters included in the design but there is even less splashing and obvious turbulence compared with all the previous filtered systems in Figure 3 . The filling times are also considerably longer but that is due to the additional volume of the running and gating. Hence the yield of these systems will be poorer than any of the systems in Figure 3.

Figure 5 clearly shows that the cusp design of runner to distribute metal between similar multiple castings is far superior to either the stepped or tapered runner systems. The entrained air volume fraction in both cusp designed is lower and the individual castings fill at approximately the same time. Essentially, by splitting the runner bar before it reaches the ingates of any casting, the liquid metal can be equally distributed and better controlled. Stepping or tapering the system just allows the metal to take the path of least resistance namely the casting at the far end of the runner where the metal can jet into the casting. Close to the complete filling of the casting it can also be seen that the curved cusp system even outperforms the straight cusp system with the lowest entrained air volume fraction of the four systems investigated. The other point to note is that in this system the castings are also much more similar in the amount of air entrained and the pattern whereas in the other systems there is considerable variation between the castings.

Comparing these relatively well-designed systems with some filling systems still in practical use (such as a horizontal ingate system and a badly-designed vertical ingate system (Figure 6a and b respectively), even BRS (which is the worst among the 10 systems) is a much better design because its offset step pouring basin entrains little air and its narrow sprue and thin runner are good enough to control the flow of metal. What's more, its wide and thin vertical ingate is also an effective device for reducing velocity before metal goes into mold cavity.

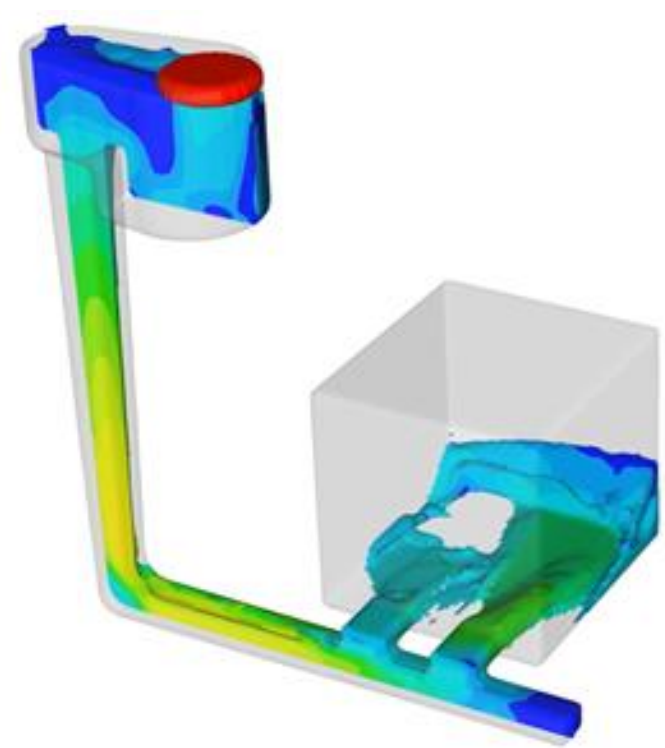

Figure 6a: Horizontal ingates (wider sprue and thicker runner compared with BRS). Metal impinges with the far end of cavity from the two horizontal ingates and causes a destructive back flow and turbulence.

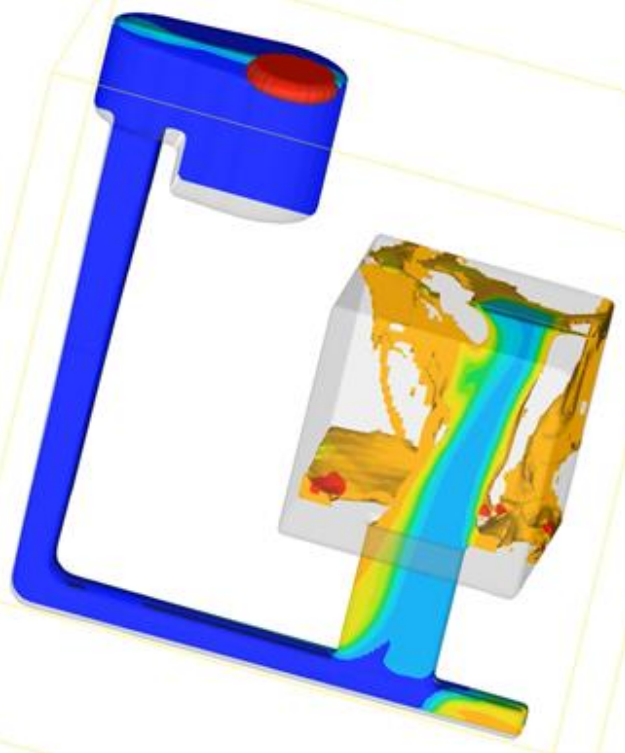

Figure 6b: Vertical ingate (wider sprue, thicker runner and narrower ingate compared with BRS). The out of control metal jets from the vertical ingate and even impacts the top face of cavity and then falls back, resulting in a completely disordered metal flow and large volume of bubbles. 


\section{Conclusions}

After detailed comparison and analysis of 10 running systems discussed in Campbell's Mini Casting Handbook [2] it is concluded that:

- $\quad$ Filters in HFS, LFS and LFS_BT all work well in reducing air entrainment;

- The bubble trap is an effective way to absorb air in the metal;

- VGS and TGS both perform well in reducing metal velocity, but filters in these two systems show little effect on reducing air entrainment, which needs more investigation;

- The best system to introduce metal into multiple ingates is the cusp junction, which distributes metal more evenly.

- The specific cusp design is important in ensuring that the casting being filled are reproducible.

- All of the running systems investigated performed better than poorly design systems with parallel rather than tapered sprue and a thick runner bar.

\section{References}

[1] J. Campbell, Complete Casting Handbook, Second ed., Oxford: Butterworth-Heinemann, 2015.

[2] J. Campbell, Mini Casting Handbook, Malvern, UK: Aspect Design, 2017.

[3] J. Campbell, Castings Practice: The Ten Rules of Casting, Oxford: Butterworth-Heinemann, 2004.

[4] M. Jolly, "Prof John Campbell's ten rules for making reliable castings," Journal of Materials, vol. 57, no. 5, pp. 19-28, 2005.

[5] S. Nafisi, B. Mosleh, G. R. Kazazie and M. A. Boutorabi, "Reviewing Aluminum Gating Systems," Modern Casting, pp. 35-39, 2010.

[6] P. D. Ingle and B. E. Narkhede, "A Literature Survey Of Methods To Study And Analyze The Gating System Design For Its Effect On Casting Quality," Materials Today, vol. 5, no. 2 Part 1, pp. 5421-5429, 2018.

[7] R. A. Miller, S. C. Lu and A. B. Rebello, "Simple visualization techniques for die casting part and die design. Final report," US Dept of Energy, Office of Scientific and Technical Information, 1997.

[8] A. Kheirabi, A. Baghani, A. Bahmani, M. Tamizifar, P. Davami, M. O. Shabani and A. Mazahery, "Understanding the occurrence of the surface turbulence in a nonpressurized bottom gating system: Numerical simulation of the melt flow pattern," Proceedings of the Institution of Mechanical Engineers: Part L: Journal of Materials: Design and Applications, vol. 232, no. 3, pp. 230-241, 17 December 2015.

[9] J. Campbell, "Sixty Years of Casting Research," Metallurgical \& Materials Transactions (A), vol. 46, no. 11, pp. 4848-4853, 2015.

[10] J. Campbell, "Stop Pouring, Start Casting," International Journal of Metalcasting, vol. 6, no. 3, pp. 7-18, 2012.

[11] Hsu, Y. F and H. J. Lin, "Foam filters used in gravity casting," Metallurgical and Materials Transactions: B, vol. 42, no. 6, pp. 1110-1117, 2011.

[12] M. R. Jolly, "Chapter 18: Castings," in Comprehensive Structural Integrity, vol. 1, I. Milne, R. O. Ritchie and B. Karihaloo, Eds., Oxford, Elsevier, 2003, pp. 377-466.

[13] S. L. Nimbulkar and R. S. Dalu, "Design optimization of gating and feeding system through simulation technique for sand casting of wear plate," Perspectives in Science, vol. 8, pp. 39-42, 2016.

[14] C. Esparza, M. Guerrero and R. Rios-Mercado, "Optimal design of gating systems by gradient search methods," Computational Materials Science, vol. 36, no. 4, pp. 457-467, 2006.

[15] H. B. H, K. K. Tong, X. P. Niu and I. Pinwill, "Design and optimisation of runner and gating systems for the die casting of thin-walled magnesium telecommunication parts through numerical simulation," Journal of Materials Processing Technology, vol. 105, no. 1-2, pp. 128-133, 2000.

[16] M. W. Fu and M. S. Yong, "Simulation-enabled casting product defect prediction in die casting process," International Journal of Production Research, vol. 47, no. 18, pp. 5203-5216, 2009.

[17] M. R. Jolly and J.-C. Gebelin, "Experimentation and numerical modeling of metal flow through filters," in Tenth International Conference on Modelling of Casting Welding and Advanced Solidification Processes X (MCWASPX), Sandestin, FA, USA, 2003. 
2019-02-09

Numerical process modelling and simulation of campbell running systems designs

\section{Lyu, Chengcheng}

Springer

Lyu C, Papanikolaou M, Jolly M. (2019) Numerical process modelling and simulation of campbell running systems designs. In: Shape Casting: The Minerals, Metals \& Materials Series, 7th International Symposium Celebrating Prof. John Campbell's 80th Birthday, pp. 53-64 https://doi.org/10.1007/978-3-030-06034-3_5

Downloaded from Cranfield Library Services E-Repository 